\title{
Stroke in prediabetic patients
}

Silva-Fernández, J; García-Ruiz, R; García-Ruiz, R; Moreno-Moreno, P; González-Pereira, C. Mancha Centro General Hospital. Alcázar de San Juan (Spain).

Objective: To describe the clinical characteristics of stroke in patients with prediabetes (pre-DM) and to compare them with diabetes mellitus (DM) and non DM patient characteristics.

Methods: Retrospective analysis of a prospective series of acute stroke patients. Demographic and clinical characteristics were compared between the three groups, along with outcome data. Stroke severity was evaluated by modified Rankin scale (mRS) and NIHSS (NIH stroke scale).

\section{RESULTS}

\begin{tabular}{|c|c|c|c|c|c|c|}
\hline Baseline data & $\begin{array}{c}\text { Non DM } \\
\text { n } 50\end{array}$ & $\begin{array}{c}\text { Pre DM } \\
\text { n } 32\end{array}$ & $\begin{array}{l}\text { DM } \\
\text { n } 56\end{array}$ & $\mathbf{p}$ & $\mathrm{p}^{*}$ & P** \\
\hline Age, median years & $74,50 \pm 15,34$ & $73,38 \pm 13,20$ & $73,84 \pm 11,44$ & ns & ns & ns \\
\hline Sex (male), n (\%) & $22(44)$ & $17(53,1)$ & $33(58,9)$ & ns & ns & ns \\
\hline Arterial Hypertensión n (\%) & $23(46)$ & $23(71)$ & $50(89)$ & $<0,05$ & ns & $<0,05$ \\
\hline Hypercholesterolemia, n (\%) & $21(42)$ & $21(65)$ & $39(69)$ & $<0,01$ & $<0,05$ & ns \\
\hline Coronary artery disease, n (\%) & $5(10)$ & $2(6,2)$ & $9(16)$ & ns & ns & ns \\
\hline Previous stroke, n (\%) & $9(18)$ & $3(9)$ & $20(35,7)$ & $<0,05$ & ns & $<0,05$ \\
\hline Peripheral artery disease, n (\%) & $2(4)$ & $2(6,2)$ & $4(7,1)$ & ns & ns & ns \\
\hline Atrial fibrillation & $3(6)$ & $5(15,6)$ & $9(16)$ & ns & ns & ns \\
\hline Current smoking, n (\%) & 7 (14) & $5(15,6)$ & $4(7,5)$ & ns & ns & ns \\
\hline Hb A1c (\%) & $5,30 \pm 0,77$ & $5,76 \pm 0,30$ & $7,50 \pm 1,87$ & $<0,001$ & ns & $<0,001$ \\
\hline
\end{tabular}

There were no differences in stroke severity between non-DM, pre-DM and DM patients $(4,9 \pm 6,2 ; 4,5 \pm 6 ; 4,91 \pm 5,4$ NIHSS median points each group), neither in 3-month Ranking scale $(2,18 \pm 2,21 ; 1,81$ $\pm 2,2 ; 1,96 \pm 1,92$ ).

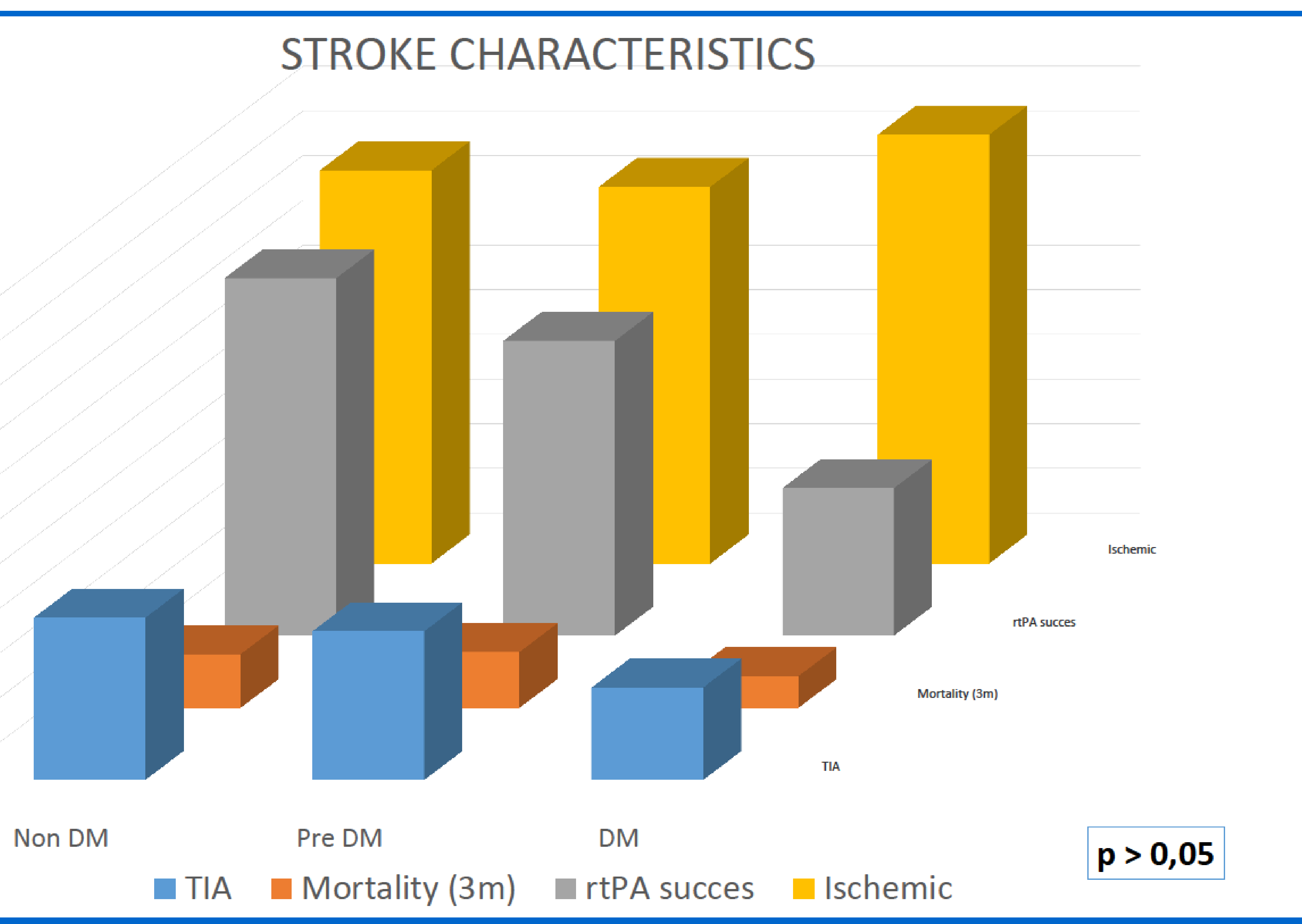

Conclusions

Prediabetes frecuency is higher than observed in general population (10\%).

Prediabetic patients occupy an intermediate situation regarding vascular risk factors (hypertension and hypercholesterolemia) but has no impact on stroke outcomes. 